\title{
Deep conservation of prion-like composition in the eukaryotic prion-former Pub1/Tial family and its relatives
}

\author{
Wan-Chun Su ${ }^{1}$, Paul M Harrison ${ }^{\text {Corresp. } 2}$ \\ 1 Dept. of Biology, McGill University, Montreal, Canada \\ 2 Dept. of Biology, McGill University, Montreal, Quebec, Canada \\ Corresponding Author: Paul M Harrison \\ Email address: paul.harrison@mcgill.ca
}

Pub1 protein is an important RNA-binding protein functional in stress granule assembly in budding yeast $S$. cerevisiae and, as its co-ortholog Tial, in humans. It is unique among proteins in evidencing prion-like aggregation in both its yeast and human forms.

Previously, we noted that Pub1/Tial was the only protein linked to human disease that has prion-like character and and has demonstrated such aggregation in both species. Thus, we were motivated to probe further into the evolution of the Pub1/Tia1 family (and its close relative Nam8 and its orthologs) to gain a picture of how such a protein has evolved over deep evolutionary time since the last common ancestor of eukaryotes. Here, we discover that the prion-like composition of this protein family is deeply conserved across eukaryotes, as is the prion-like composition of its close relative Nam8/Ngr1. A sizeable minority of protein orthologs have multiple prion-like domains within their sequences (6-20\% depending on criteria). The number of RNA-binding RRM domains is conserved at three copies over $>86 \%$ of the Pub1 family ( $>71 \%$ of the Nam8 family), but proteins with just one or two RRM domains occur frequently in some clades, indicating that these are not due to annotation errors. Overall, our results indicate that a basic scaffold comprising three RNA-binding domains and at least one prion-like region has been largely conserved since the last common ancestor of eukaryotes, providing further evidence that prion-like aggregation may be a very ancient and conserved phenomenon for certain specific proteins. 
1

2 Deep conservation of prion-like composition in the eukaryotic prion-

3 former Pub1/Tia1 family and its relatives

4

5 Wan-Chun $\mathrm{Su}^{1}$ \& Paul Martin Harrison ${ }^{1}$

6

$7{ }^{1}$ Department of Biology,

8 McGill University,

9 Montreal, QC,

10 Canada.

11

12 Corresponding author:

13 Paul Harrison

14 Email: paul.harrison@mcgill.ca

15

16

17 
18

19

\section{Abstract:}

Pub1 protein is an important RNA-binding protein functional in stress granule assembly in budding yeast $S$. cerevisiae and, as its co-ortholog Tia1, in humans. It is unique among proteins in evidencing prion-like aggregation in both its yeast and human forms. Previously, we noted that Pub1/Tial was the only protein linked to human disease that has prion-like character and has demonstrated such aggregation in both species. Thus, we were motivated to probe further into the evolution of the Pub1/Tia1 family (and its close relative Nam8 and its orthologs) to gain a picture of how such a protein has evolved over deep evolutionary time since the last common ancestor of eukaryotes. Here, we discover that the prion-like composition of this protein family is deeply conserved across eukaryotes, as is the prion-like composition of its close relative Nam8/Ngr1. A sizeable minority of protein orthologs have multiple prion-like domains within their sequences (6$20 \%$ depending on criteria). The number of RNA-binding RRM domains is conserved at three copies over $>86 \%$ of the Pub1 family ( $>71 \%$ of the Nam8 family), but proteins with just one or two RRM domains occur frequently in some clades, indicating that these are not due to annotation errors. Overall, our results indicate that a basic scaffold comprising three RNA-binding domains and at least one prion-like region has been largely conserved since the last common ancestor of eukaryotes, providing further evidence that prion-like aggregation may be a very ancient and conserved phenomenon for certain specific proteins.

\section{Introduction}

Prions in eukaryotes have been linked to diseases, evolutionary capacitance, large-scale genetic control and long-term memory formation. Prion formation and propagation have been studied extensively in the budding yeast Saccharomyces cerevisiae. The yeast $S$. cerevisiae has 
$41>200$ prion-like proteins that have N/Q-rich domains of the sort observed in $>10$ known prion-

42 formers (An et al. 2016). A domain of the yeast protein Pub1 exhibits prion-like protein 43 aggregation and propagation in S. cerevisiae (Alberti et al. 2009). Pub1 in S. cerevisiae is a poly44 (A)+-binding protein that functions in stress granule assembly, and is required for mRNA stability 45 for many transcripts (Anderson et al. 1993; Matunis et al. 1993; Rayman \& Kandel 2017). Pub1 46 protein abundance is linked to increased DNA replication stress (Tkach et al. 2012). There is 47 evidence that the N/Q-rich prion-like domain of Pub1 is functional in recruiting protein synthesis machinery to the cytoskeleton, which is critical for maintaining cytoskeletal integrity (Li et al. 2014). In doing so, Pub1 interacts with the [PSI+]-prion-forming Sup35 protein to form a twoprotein self-propagating state (Li et al. 2014). Pub1 can act as an accessory translation factor that appears to fine-tune translation termination efficiency (Urakov et al. 2017). Similarly to Sup35 (Franzmann et al. 2018), Pub1 forms condensates upon cellular stress (Kroschwald et al. 2018). Condensates induced by different stresses have different biophysical characteristics, that is, some are gel-like, while others are more solid-like (Kroschwald et al. 2018). Another dimension to the relationship between Sup35 and Pub1 is that the latter appears to be involved in a cellular mechanism to alleviate toxicity of the $[\mathrm{PSI}+]$ prion, which is formed from Sup35 proteins (Urakov et al. 2018). Pub1 is one of a small number of prion-formers that serve as protein interaction hubs for other prion-like proteins (Harbi \& Harrison 2014b). The Pub1 prion-forming domain is one of the most rapidly evolving prion-forming domains sequence-wise (Su \& Harrison 2019). The Pub1 paralog Nam8 (which also contains a prion-like domain) is a part of the U1 snRNP protein 61 complex; mutants of Nam8 are defective in meiotic recombination (Puig et al. 1999; Qiu et al. 2011). Nam8 was one of a small panel of genes that were discovered in a genome-wide genetic screen of yeast to rescue the cytotoxicity of FUS/TLS, a prion-like protein that causes some 
64 familial amyotrophic lateral scleroses (Ju et al. 2011). Nam8 is non-essential in the U1 snRNP

65 protein complex, but demonstrates synthetic-lethal genetic interactions with other non-essential 66 interactors (Agarwal et al. 2019).

67 The Pub1 human homolog, Tia1, is also functional through prion-like aggregation in stress 68 granule assembly in mammalian cells (Gilks et al. 2004; Rayman \& Kandel 2017). The prion-like 69 domain of Tia 1 can be substituted with that of Sup35 and still maintain its stress granule assembly 70 role, and forms protease-resistant aggregates in mammalian cells (Gilks et al. 2004). Full-length 71 Tial can form self-perpetuating prion-like aggregates heterologously in S. cerevisiae (Li et al. 72 2014). Tial and its paralog Tiar (also called TiaL1) together regulate stress response and cell-cycle 73 progression mediated by the protein kinase EIF2AK2 (Meyer et al. 2018). Reduction of Tia1 levels 74 protects against tau-mediated neurodegeneration in the brain, rescues behavioral deficits and 75 improves survival (Apicco et al. 2018). Single-nucleotide polymorphisms (SNPs) in Tial are 76 linked at very low frequency to amyotrophic lateral sclerosis and frontotemporal dementia in 77 Chinese cohorts but not in European ones (Baradaran-Heravi \& Consortium 2018; Gu et al. 2018; Zhang et al. 2018). SNPs in Tia1 are linked to a multisystem proteinopathy that involves disruption of stress granule formation (Lee et al. 2018), and to Welander distal myopathy (Hackman et al. 2013; Klar et al. 2013).

All of these proteins (Pub1, Nam8, etc.) are examples of proteins that contain RNA-binding RRM domains. The RRM domain provides RNA-binding functionality for a diverse set of proteins across the eukaryotic clade, including dozens in S. cerevisiae. It comprises a very conserved alphabeta protein fold that can bind to a large number of diverse RNA sequences (Daubner et al. 2013). Previously, we noted that Pub1/Tia1 was the only protein linked to human disease that has prion-like character and aggregation in both humans and S. cerevisiae (An \& Harrison 2016). Here, 
87 motivated by these observations, we have probed further into the evolution of the Pub1/Tial

88 family, including the close relative Nam8 and its orthologs. We discover that prion-like

89 composition is deeply conserved across a wide diversity of eukaryotes, with some clades of

90 proteins conserving multiple prion-like domains within their individual sequences.

\section{Methods}

93 Data

94 Sets of orthologs for the following protein families were downloaded from OrthoDB

95 database version 10.1 at the Eukaryota level (Kriventseva et al. 2019): Pub1/Tia1 (OrthoDB group

96 1066369at2579), Nam8/Ngr1 (group 775799at2579) and Pab1 (group 1027234at2759). These

97 data also contain paralogs. In addition, lists of reciprocal-best-hits (RBH) orthologs were made for

98 the same proteins across sets of reference eukaryotic proteomes downloaded from UniProt version

99 2019_1 (UniProt 2019). These were cross-referenced with the OrthoDB sets to determine the

100 subset of OrthoDB annotations that are such RBH orthologs. Also, only OrthoDB proteins that are

101 from the UniProt reference proteomes were considered for further analysis. FASTA-format files

102 of the Pub1/Tia1/Tiar and Nam8/Ngr1 families of proteins are provided as supplementary data 103 (Data S1 \& S2).

105 Multiple sequence alignment and phylogenetic trees

106 Multiple sequence alignments were constructed using KMAD (Lange et al. 2016), using

107 default parameters. KMAD is designed to handle sequences with intrinsic disorder, a trait expected 108 characteristically of prion-like proteins (Harbi \& Harrison 2014b). Phylogenetic trees were made 109 using PHYML with Bayesian information criterion and aBayes branch support (Guindon et al. 
110 2005), with the exception of a tree including extra paralogs described below. The aBayes branch

111 support is a Bayesian-like transformation of the standard approximate likelihood ratio test

112 implemented in PhyML, that is very fast and has high statistical power (Anisimova et al. 2011).

113 To construct a phylogenetic tree with extra paralogs, we used a stepwise procedure of tree

114 construction. Examination of BLASTP (Altschul et al. 1997) output indicates that Pab1 and its

115 orthologs were the next most similar RRM-domain-containing proteins in yeast that are widely

116 conserved across eukaryotes (Table S3). Pab1 is a polyadenylate-binding protein that is an

117 important interactor at various stages of messenger mRNA stability, biogenesis and translation.

118 We used similarities to Pab1 to help us to filter for further paralogs to the Pub1/Tial and

119 Nam8/Ngr1 proteins as follows. BLASTP was used to compare each ortholog from the Pub1/Tia1,

$120 \mathrm{Nam8/Ngr1}$ and Pab1 protein sets against their own proteomes, and any significant matches (with

121 e-value $\leq 1 \mathrm{e}-04)$ against the majority $(>50 \%$ length) of a proteome sequence that matched a

122 Pub1/Tia1 or Nam8/Ngr1 sequence in preference to a Pab1 sequence were labelled as potential

123 paralogs of Pub1/Tia1 or Nam8/Ngr1, formed more recently in specific eukaryotic clades. Then,

124 a phylogenetic tree was constructed using the RaxML program (using the PROTGAMMAJTTF

125 mutation parameters and the ' $\mathrm{f}$ a' flag for rapid bootstrap analysis and 100 bootstrap replicates)

126 (Stamatakis 2015) with the Pub1/Tia1, Nam8/Ngr1 and Pab1 ortholog sets plus the additional

127 potential paralogs proteins as input. The tree was pruned at an appropriate node to remove all of

128 the Pab1 sequences and associated paralogs to leave just those paralogs that were formed more

129 recently than the last common ancestor of the Pub1/Tia1 and Nam8 proteins.

130

131 Annotation of RNA-binding (RRM) domains 
13366.0 from the database Pfam (El-Gebali et al. 2019; Mitchell et al. 2019). We checked these

134 annotations against those also for the PROSITE and SMART databases (Letunic \& Bork 2018;

135 Sigrist et al. 2010), and they differ by $<1 \%$ of extra or fewer domain annotations, which gave us

136 confidence to proceed with the Pfam set. Since there were some OrthoDB sequences that do not

137 have Pfam annotations, and other annotations may be incomplete, we generated additional RRM

138 domain annotations. Firstly, the sequences for the RRM domain annotations were extracted and

139 then compared against the ASTRAL SCOPe database version 2.07 (Fox et al. 2014) using

140 BLASTP (Altschul et al. 1997) with e-value threshold 1e-04, to pull out the identities of protein

141 domain structures that are for RRM domains. Then the ASTRAL SCOPe protein domains plus the

142 sequence fragments for the RRM-domain annotations were then formed into a BLASTP database

143 to compare against the OrthoDB sequences to augment the existing list of RRM-domain

144 annotations (again using e-value threshold 1e-04). These were then reduced for overlap by sorting

145 them in decreasing order of domain length and progressively flagging overlappers further down

146 the list for deletion.

\section{Annotation of prion-like domains and intrinsic disorder}

Prion-like domains were annotated using the PLAAC program with default parameters

150 (Lancaster et al. 2014). Prion-like domains were counted using two thresholds for the PLAAC log

151 likelihood ratio (LLR) score: $>0.0$ and $\geq 15.0$. Any score $>0.0$ indicates some predicted propensity

152 to form a prion-like domain; the second threshold of 15.0 was picked since the lowest LLR value

153 for a known fungal prion-forming domain that was picked oput for experimental analysis is $\sim 16.0$

154 in the fission yeast prion-former Ctr4 (Sideri et al. 2017). The program fLPS was used to detect 
155 compositional bias for glutamine and asparagine residues, which is a bias characteristic of the

156 substantial majority of the known prion-forming domains in budding yeast and other organisms

157 (Harrison 2006; Harrison 2017). Expected fractional frequencies $=0.05$ for both glutamine and

158 asparagine were used in running fLPS. fLPS Binomial P-values indicate the degree of

159 compositional bias, and here we picked the most biased subsequence for either $\mathrm{Q}$ or $\mathrm{N}$ or $\mathrm{Q}+\mathrm{N}$ for

160 each sequence. The $\log 10$ of these P-values is investigated here for all the data analysis.

161 To examine for multiple prion-like domains, the sequences were split up into fragments

162 with the ends delimited by RRM domains and other protein domains (plus a 10 amino-acid-residue

163 buffer added onto both ends), which were then input into the PLAAC program. Prion-forming

164 N/Q-rich domains are highly intrinsically disordered (Harbi \& Harrison 2014b). Intrinsic disorder

165 was annotated using the IUPRED2a and DISOPRED3 programs with default parameters

166 (Dosztanyi 2018; Ward et al. 2004). The total percentage of intrinsic disorder was calculated for

167 each whole protein sequence, since there is not an intrinsic disorder score for a specific tract that

168 can be extracted from the output of the disorder annotation programs. Intrinsic disorder

169 assignments by IUPRED2a and DISOPRED3 are strongly correlated $(\mathrm{R}=0.43$ for Pub1, $\mathrm{R}=0.47$

170 for Nam8, P-values <1e-20).

\section{Results}

\section{Evolution within Saccharomycetes}

174 In Saccharomycetes, the three main proteins from the protein families under study are

175 Pub1, Nam8 and Ngr1. Preliminary analysis of phylogenetic trees indicated that Ngr1 formed by

176 duplication from Nam8 in the last common ancestor of Saccharomycetes. Firstly, we assessed the

177 conservation of prion-like composition across the Saccharomycetes clade for Pub1, and for its 
178 paralogs Nam8 and Ngr1. Prion-like composition is largely maintained for all three paralogs across

179 this clade (Figure 1). The substantial majority of Pub1 orthologs have PLAAC prion-like region

180 scores $\geq 15.0(53 / 65,82 \%)$, and almost all $>0.0(61 / 65,94 \%)$; less so for Nam8 and Ngr1 $(73 / 114$,

$18164 \% \geq 15.0 ; 113 / 114,99 \%>0.0)$.

182

183

\section{Evolution across eukaryotes}

Furthermore, this prion-like composition is deeply conserved across large diverse clades

within the whole eukaryotic domain for the Pub1/Tial and Nam8/Ngr1 families (Figure 2).

Overall, $90 \%$ of sequences have PLAAC $\log$ likelihood ratio (LLR) scores $>0.0$, with $58 \% \geq 15.0$.

Specifically for Pub1, $84 \%$ have scores $>0.0$ and $60 \%$ have scores $\geq 15.0$. Prion-forming N/Q-rich domains are highly intrinsically disordered (Harbi \& Harrison 2014b). There is some degree of correlation of LLR score with overall percentage annotated intrinsic disorder in the sequences, particularly for Pub1 ( $\mathrm{P}<2 \mathrm{e}-16$; Figure 3B), but some clades on the phylogenetic tree are observed that have low prion-like character and relatively high intrinsic disorder (Figure 2).

for both the Pub1/Tia1 and Nam8/Ngr1 families (Figure 3A). PLAAC LLR scores are strongly correlated with the fLPS compositional biases for $\mathrm{Q}$ and $\mathrm{N}$ residues $(\mathrm{R}=0.70$ for $\mathrm{Nam} 8$, and $\mathrm{R}=0.52 ; \mathrm{P}<1 \mathrm{e}-20$ ), but substantial clades can be seen on the phylogenetic tree that have low fLPS $\mathrm{Q} / \mathrm{N}$ residue bias, but high PLAAC LLR scores, such as some of the fungal clades clustering near Saccharomycetes.

The larger tree with additional paralogs whose construction is described in Methods,

199 indicates that no further major paralogs have formed during eukaryotic speciation, since these

200 would appear as intermittent larger blocks in the tree figure (Figure S1). Such paralogs have 
201 however formed sporadically in evolutionarily more recent clades; some of these appear to have

202 lost the prion-like domains, or intrinsic disorder generally (Figure S1).

203

204 Multiple prion-like domains occur in several diverse clades

205

206

207

208

209

210

211

212

213

214

215

216

217

218

219

220

221

222

Prion-forming proteins are usually characterized as having one distinct prion-forming area (Harbi \& Harrison 2014a; Harbi et al. 2012). Likewise, prion-like proteins are usually annotated by picking out a single region with the highest adjudged propensity for forming prions; the algorithms that have been derived for annotating prion-like proteins are all based on this principle (Harrison 2017; Lancaster et al. 2014; Ross et al. 2013; Zambrano et al. 2015). However, it is possible that multiple prion-forming regions can occur within the same protein chain. We decided to check for this in the protein families under study here, since from spot-checking they often contain more than one annotated intrinsically disordered region delimited by the structured protein domains (specifically here most often the RRM RNA-binding domain). We parsed each protein sequence into fragments delimited by the positions of protein domains and annotated these fragments for prion-like composition, as described in Methods. We find that it is not uncommon to have multiple prion-like regions in one protein chain, particulary in the Nam8/Ngr 1 family $(11 \%$ $\geq 2$ prion-like regions for the Nam8/Ngr1 family and 1\% for the Pub1/Tia1 family, Figure 4B-C). Indeed, there are specific large clades that maintain two prion-like regions (Figure 2). Only with a lower PLAAC LLR score threshold of $>0.0$ does the Pub1/Tia1 family display a sizeable minority of protein sequences with multiple prion-like regions ( $10 \%$ with $\geq 2$ prion-like domains; Figures 2 and 4B-C). A large fraction of these extra domains with prion-like composition are internal in the sequence (46\% for Pub1/Tia1 and 29\% for Nam8/Ngr1 for PLAAC LLR threshold $>0.0$ ).

Peer) reviewing PDF | (2019:10:42587:1:1:NEW 16 Mar 2020) 
The number of RNA-binding RRM domains is largely fixed at three for both the sequences having $>3$ ), but proteins with just one or two RRM domains occur frequently in some specific clades (Figures 2 and 4A).

\section{Discussion}

231

232

233

234 235

\section{Evolution across eukaryotes}

Analysis of the evolution across eukaryotes of prion-like composition for the Pub1/Tia1 and Nam8 families, as judged by the PLAAC program [ref], indicated that such composition is deeply conserved. We can therefore surmise that this composition is important for the protein function. However there are some clades that: (i) have low prion-like character and maintain relatively high annotated intrinsic disorder; (ii) have high prion-like character as judged by PLAAC [ref], but low bias for N and Q residues, as judged by the fLPS algorithm [ref]. Sequences from such clades may be good candidates for checking for alternative compositions for prion-like domains in Pub1 that fulfil either its prion-forming ability or its role in stress granule formation.

Sequences of type (ii) above would particularly be interesting candidates for further experimental study for prion-like aggregation, since the prion-like composition is made up more from non- $\mathrm{Q}+\mathrm{N}$ residues that contribute to prion-forming biases in the known $S$. cerevisiae cases.

\section{Multiple prion-like domains occur in several diverse clades}


The occurrence of multiple prion-like domains in proteins raises the possibility of competing prion-like aggregation by the different prion-like domains within the same protein sequence. However, a large fraction of these extra domains with prion-like composition are 248 internal in the sequence, indicating that they would have greater difficulty forming amyloid fibrils. 249 Also, multiple prion-like domains might affect the character of intracellular condensates, i.e., as 250 either solid-like or gel-like, perhaps making them more solid-like because of the increased prion251 like composition relative to other types of intrinsic disorder propensity in the equivalent regions 252 in other orthologs (Kroschwald et al. 2018).

\section{The Number of RNA-binding domains is largely fixed at three}

We found very strong conservation of a three-domain RRM RNA-binding domain pattern frequently in a conserved pattern in some smaller clades, which indicates that these are not due to annotation errors and are real evolutionary trends (Figures 2 and 4A). There are a handful of sequences with four or more RRM domains, but it is unclear whether these may be annotation errors, due to their sporadic occurrence (Figure 2). One notable cluster that has either as one or two RRM domains (upper left corner of Figure 2, near clade labelled with blue band), consists mainly of vertebrate Nam8 orthologs that are assigned function in selenocysteine biosynthesis, 
268 development of muscles, skin, the nervous system and immune system (Schweizer \& Fradejas-

269 Villar 2016). These proteins also tend to have lower annotated intrinsic disorder content and lower

270 prion-like composition (Figure 2).

271

272 Conclusions

273

Previously, we noted that Pub1/Tial was unique in being linked to human disease while

274 having prion-like character aggregation in both humans and the budding yeast $S$. cerevisiae. Thus,

275 we were motivated to probe further into the evolution of the Pub1/Tial family and its close

276 relatives, to gain a picture of how such a protein has evolved over deep evolutionary time since

277 the last common ancestor of eukaryotes. We discovered that the prion-like composition of the

278 Pub1/Tia1 prion-former family, and its closely related Nam8/Ngr1 family is widely conserved

279 across eukaryotes, with $90 \%$ of sequences having PLAAC LLR scores $>0.0$, with $58 \% \geq 15.0$. Also,

280 the number of RNA-binding domains in these sequences is widely conserved, with $70 \%$ of these

281 sequences having 3 RRM domains. A sizeable minority of the proteins in these ortholog families

282 contain multiple prion-like domains within them, particularly for certain clades of the Nam8/Ngr1

283 family ( $\sim 11 \%$ of this family having $\geq 2$ prion-like domains with PLAAC LLR scores $\geq 15.0$ ).

284 Overall, these results suggest that a basic scaffold comprising three RNA-binding domains

285 and at least one prion-like region has been widely conserved since the last common ancestor of

286 eukaryotes, and provide some support for the notion that prion-like aggregation may be an

287 anciently conserved phenomenon for certain specific proteins.

288

289 Acknowledgements 
This research was funded completely by the Natural Sciences Engineering Research

291 Council of Canada, except that some of the calculations were performed on a desktop computer

292 funded by both the Canada Foundation for Innovation and the Natural Sciences Engineering

293 Research Council of Canada. Computing resources supplied by Compute Canada were also used. 294

295 References

296 Agarwal R, Schwer B, and Shuman S. 2019. Domain Requirements and Genetic Interactions of the Mud1 Subunit of the Saccharomyces cerevisiae U1 snRNP. G3 (Bethesda) 9:145-151. $10.1534 / \mathrm{g} 3.118 .200781$

Alberti S, Halfmann R, King O, Kapila A, and Lindquist S. 2009. A systematic survey identifies prions and illuminates sequence features of prionogenic proteins. Cell 137:146-158.

Altschul SF, Madden TL, Schaffer AA, Zhang J, Zhang Z, Miller W, and Lipman DJ. 1997. Gapped BLAST and PSI-BLAST: a new generation of protein database search programs. Nucleic Acids Res 25:3389-3402. 10.1093/nar/25.17.3389

An L, Fitzpatrick D, and Harrison PM. 2016. Emergence and evolution of yeast prion and prionlike proteins. BMC Evol Biol 16:24. 10.1186/s12862-016-0594-3

An L, and Harrison PM. 2016. The evolutionary scope and neurological disease linkage of yeastprion-like proteins in humans. Biol Direct 11:32. 10.1186/s13062-016-0134-5

Anderson JT, Paddy MR, and Swanson MS. 1993. PUB1 is a major nuclear and cytoplasmic polyadenylated RNA-binding protein in Saccharomyces cerevisiae. Mol Cell Biol 13:6102-6113. 10.1128/mcb.13.10.6102 
311 Anisimova M, Gil M, Dufayard JF, Dessimoz C, and Gascuel O. 2011. Survey of branch support

312 methods demonstrates accuracy, power, and robustness of fast likelihood-based approximation schemes. Syst Biol 60:685-699. 10.1093/sysbio/syr041

314

315

316

317

318

319

320

321

322

323

324

325

326

327

328

329

330

331

Apicco DJ, Ash PEA, Maziuk B, LeBlang C, Medalla M, Al Abdullatif A, Ferragud A, Botelho E, Ballance HI, Dhawan U, Boudeau S, Cruz AL, Kashy D, Wong A, Goldberg LR, Yazdani N, Zhang C, Ung CY, Tripodis Y, Kanaan NM, Ikezu T, Cottone P, Leszyk J, Li H, Luebke J, Bryant CD, and Wolozin B. 2018. Reducing the RNA binding protein TIA1 protects against tau-mediated neurodegeneration in vivo. Nat Neurosci 21:72-80.

$10.1038 / \mathrm{s} 41593-017-0022-\mathrm{Z}$

Baradaran-Heravi Y, and Consortium BCEE. 2018. No supportive evidence for TIA1 gene mutations in a European cohort of ALS-FTD spectrum patients. Neurobiology of Aging 69:293.e299-293.e211.

Daubner GM, Clery A, and Allain FH. 2013. RRM-RNA recognition: NMR or crystallography...and new findings. Curr Opin Struct Biol 23:100-108. 10.1016/j.sbi.2012.11.006

Dosztanyi Z. 2018. Prediction of protein disorder based on IUPred. Protein Sci 27:331-340. $10.1002 /$ pro. 3334

El-Gebali S, Mistry J, Bateman A, Eddy SR, Luciani A, Potter SC, Qureshi M, Richardson LJ, Salazar GA, Smart A, Sonnhammer ELL, Hirsh L, Paladin L, Piovesan D, Tosatto SCE, and Finn RD. 2019. The Pfam protein families database in 2019. Nucleic Acids Res 47:D427-D432. 10.1093/nar/gky995 
332 Fox NK, Brenner SE, and Chandonia JM. 2014. SCOPe: Structural Classification of Proteins--

333

334

335

336

337

338

339

340

341

342

343

344

345

346

347

348

349

350

351

352 extended, integrating SCOP and ASTRAL data and classification of new structures. Nucleic Acids Res 42:D304-309. 10.1093/nar/gkt1240

Franzmann TM, Jahnel M, Pozniakovsky A, Mahamid J, Holehouse AS, Nuske E, Richter D, Baumeister W, Grill SW, Pappu RV, Hyman AA, and Alberti S. 2018. Phase separation of a yeast prion protein promotes cellular fitness. Science 359. 10.1126/science.aao5654

Gilks N, Kedersha N, Ayodele M, Shen L, Stoecklin G, Dember LM, and Anderson P. 2004. Stress granule assembly is mediated by prion-like aggregation of TIA-1. Mol Biol Cell 15:5383-5398. 10.1091/mbc.e04-08-0715

Gu X, Chen Y, Wei Q, Cao B, Ou R, Yuan X, Hou Y, Zhang L, Liu H, Chen X, and Shang HF. 2018. Mutation screening of the TIA1 gene in Chinese patients with amyotrophic lateral sclerosis/frontotemporal dementia. Neurobiol Aging 68:161 e161-161 e163. 10.1016/j.neurobiolaging.2018.04.010

Guindon S, Lethiec F, Duroux P, and Gascuel O. 2005. PHYML Online--a web server for fast maximum likelihood-based phylogenetic inference. Nucleic Acids Res 33:W557-559. 10.1093/nar/gki352

Hackman P, Sarparanta J, Lehtinen S, Vihola A, Evila A, Jonson PH, Luque H, Kere J, Screen M, Chinnery PF, Ahlberg G, Edstrom L, and Udd B. 2013. Welander distal myopathy is caused by a mutation in the RNA-binding protein TIA1. Ann Neurol 73:500-509. 10.1002/ana.23831

Harbi D, and Harrison PM. 2014a. Classifying prion and prion-like phenomena. Prion 8. 
353 Harbi D, and Harrison PM. 2014b. Interaction networks of prion, prionogenic and prion-like 354 proteins in budding yeast, and their role in gene regulation. PLoS One 9:e100615. 10.1371/journal.pone.0100615

356

357

358

359

360

361

362

363

364

365

366

367

368

369

370

371

372

373

374

Harbi D, Parthiban M, Gendoo DM, Ehsani S, Kumar M, Schmitt-Ulms G, Sowdhamini R, and Harrison PM. 2012. PrionHome: a database of prions and other sequences relevant to prion phenomena. PLoS One 7:e31785.

Harrison PM. 2006. Exhaustive assignment of compositional bias reveals universally prevalent biased regions: analysis of functional associations in human and Drosophila. $B M C$ Bioinformatics 7:441. 10.1186/1471-2105-7-441

Harrison PM. 2017. fLPS: Fast discovery of compositional biases for the protein universe. $B M C$ Bioinformatics 18:476. 10.1186/s12859-017-1906-3

Ju S, Tardiff DF, Han H, Divya K, Zhong Q, Maquat LE, Bosco DA, Hayward LJ, Brown RH, Jr., Lindquist S, Ringe D, and Petsko GA. 2011. A yeast model of FUS/TLS-dependent cytotoxicity. PLoS Biol 9:e1001052. 10.1371/journal.pbio.1001052

Klar J, Sobol M, Melberg A, Mabert K, Ameur A, Johansson AC, Feuk L, Entesarian M, Orlen H, Casar-Borota O, and Dahl N. 2013. Welander distal myopathy caused by an ancient founder mutation in TIA1 associated with perturbed splicing. Hum Mutat 34:572-577. 10.1002/humu.22282

Kriventseva EV, Kuznetsov D, Tegenfeldt F, Manni M, Dias R, Simao FA, and Zdobnov EM. 2019. OrthoDB v10: sampling the diversity of animal, plant, fungal, protist, bacterial and viral genomes for evolutionary and functional annotations of orthologs. Nucleic Acids Res 47:D807-D811. 10.1093/nar/gky1053 
375 Kroschwald S, Munder MC, Maharana S, Franzmann TM, Richter D, Ruer M, Hyman AA, and 376 Alberti S. 2018. Different Material States of Pub1 Condensates Define Distinct Modes of 377 Stress Adaptation and Recovery. Cell Rep 23:3327-3339. 10.1016/j.celrep.2018.05.041

378 Lancaster AK, Nutter-Upham A, Lindquist S, and King OD. 2014. PLAAC: a web and 379 command-line application to identify proteins with prion-like amino acid composition. Bioinformatics 30:2501-2502. 10.1093/bioinformatics/btu310

381 Lange J, Wyrwicz LS, and Vriend G. 2016. KMAD: knowledge-based multiple sequence 382 alignment for intrinsically disordered proteins. Bioinformatics 32:932-936. 10.1093/bioinformatics/btv663

Lee Y, Jonson PH, Sarparanta J, Palmio J, Sarkar M, Vihola A, Evila A, Suominen T, Penttila S, 385 Savarese M, Johari M, Minot MC, Hilton-Jones D, Maddison P, Chinnery P, Reimann J, 386 Kornblum C, Kraya T, Zierz S, Sue C, Goebel H, Azfer A, Ralston SH, Hackman P, Bucelli RC, Taylor JP, Weihl CC, and Udd B. 2018. TIA1 variant drives myodegeneration in multisystem proteinopathy with SQSTM1 mutations. J Clin Invest 128:1164-1177. 10.1172/JCI97103

Letunic I, and Bork P. 2018. 20 years of the SMART protein domain annotation resource.

392 Letunic I, and Bork P. 2019. Interactive Tree Of Life (iTOL) v4: recent updates and new 393 developments. Nucleic Acids Res 47:W256-W259. 10.1093/nar/gkz239

394 Li X, Rayman JB, Kandel ER, and Derkatch IL. 2014. Functional role of Tia1/Pub1 and Sup35 395 prion domains: directing protein synthesis machinery to the tubulin cytoskeleton. Mol Cell 55:305-318. 10.1016/j.molcel.2014.05.027 
397 Matunis MJ, Matunis EL, and Dreyfuss G. 1993. PUB1: a major yeast poly(A)+ RNA-binding $398 \quad$ protein. Mol Cell Biol 13:6114-6123. 10.1128/mcb.13.10.6114

399 Meyer C, Garzia A, Mazzola M, Gerstberger S, Molina H, and Tuschl T. 2018. The TIA1 RNA400 Binding Protein Family Regulates EIF2AK2-Mediated Stress Response and Cell Cycle 401 Progression. Mol Cell 69:622-635 e626. 10.1016/j.molcel.2018.01.011

402

403

404

405

406

407

408

409

410

411

412

413

414

415

416

417

418

419

Gebali S, Fraser MI, Gough J, Haft DR, Huang H, Letunic I, Lopez R, Luciani A, Madeira F, Marchler-Bauer A, Mi H, Natale DA, Necci M, Nuka G, Orengo C, Pandurangan AP, Paysan-Lafosse T, Pesseat S, Potter SC, Qureshi MA, Rawlings ND, Redaschi N, Richardson LJ, Rivoire C, Salazar GA, Sangrador-Vegas A, Sigrist CJA, Sillitoe I, Sutton GG, Thanki N, Thomas PD, Tosatto SCE, Yong SY, and Finn RD. 2019. InterPro in 2019: improving coverage, classification and access to protein sequence annotations. Nucleic Acids Res 47:D351-D360. 10.1093/nar/gky1100

Puig O, Gottschalk A, Fabrizio P, and Seraphin B. 1999. Interaction of the U1 snRNP with nonconserved intronic sequences affects 5' splice site selection. Genes Dev 13:569-580. 10.1101/gad.13.5.569

Qiu ZR, Schwer B, and Shuman S. 2011. Defining the Mer1 and Nam8 meiotic splicing regulons by cDNA rescue. $R N A$ 17:1648-1654. 10.1261/rna.2792011

Rayman JB, and Kandel ER. 2017. TIA-1 Is a Functional Prion-Like Protein. Cold Spring Harb Perspect Biol 9. 10.1101/cshperspect.a030718

Ross ED, Maclea KS, Anderson C, and Ben-Hur A. 2013. A bioinformatics method for identifying Q/N-rich prion-like domains in proteins. Methods Mol Biol 1017:219-228. 10.1007/978-1-62703-438-8_16 
420 Schweizer U, and Fradejas-Villar N. 2016. Why 21? The significance of selenoproteins for

421 human health revealed by inborn errors of metabolism. FASEB J 30:3669-3681.

422 $10.1096 /$ fj.201600424

423 Sideri T, Yashiroda Y, Ellis DA, Rodriguez-Lopez M, Yoshida M, Tuite MF, and Bahler J.

424 2017. The copper transport-associated protein Ctr4 can form prion-like epigenetic

425

426 determinants in Schizosaccharomyces pombe. Microb Cell 4:16-28.

427

428

Sigrist CJ, Cerutti L, de Castro E, Langendijk-Genevaux PS, Bulliard V, Bairoch A, and Hulo N. $10.15698 / \mathrm{mic} 2017.01 .552$

429 2010. PROSITE, a protein domain database for functional characterization and annotation. Nucleic Acids Res 38:D161-166. 10.1093/nar/gkp885

Small-Howard A, Morozova N, Stoytcheva Z, Forry EP, Mansell JB, Harney JW, Carlson BA, 431 Xu XM, Hatfield DL, and Berry MJ. 2006. Supramolecular complexes mediate selenocysteine incorporation in vivo. Mol Cell Biol 26:2337-2346. 10.1128/MCB.26.6.2337-2346.2006

Stamatakis A. 2015. Using RAxML to Infer Phylogenies. Curr Protoc Bioinformatics 51:6 14 11-14. 10.1002/0471250953.bi0614s51

Su TY, and Harrison PM. 2019. Conservation of Prion-Like Composition and Sequence in Prion-

Subramanian B, Gao S, Lercher MJ, Hu S, and Chen WH. 2019. Evolview v3: a webserver for visualization, annotation, and management of phylogenetic trees. Nucleic Acids Res 47:W270-W275. 10.1093/nar/gkz357 
442 Tkach JM, Yimit A, Lee AY, Riffle M, Costanzo M, Jaschob D, Hendry JA, Ou J, Moffat J,

443 Boone C, Davis TN, Nislow C, and Brown GW. 2012. Dissecting DNA damage response

444 pathways by analysing protein localization and abundance changes during DNA

445 replication stress. Nat Cell Biol 14:966-976. 10.1038/ncb2549

446 UniProt C. 2019. UniProt: a worldwide hub of protein knowledge. Nucleic Acids Res 47:D506-

447

D515. 10.1093/nar/gky1049

448 Urakov VN, Mitkevich OV, Dergalev AA, and Ter-Avanesyan MD. 2018. The Pub1 and Upf1

449

Proteins Act in Concert to Protect Yeast from Toxicity of the [PSI(+)] Prion. Int J Mol

450

Sci 19. 10.3390/ijms19113663

451

452

453

Urakov VN, Mitkevich OV, Safenkova IV, and Ter-Avanesyan MD. 2017. Ribosome-bound Pub1 modulates stop codon decoding during translation termination in yeast. FEBS J

454

455

456

457

458

459

460

461

462

463

464

284:1914-1930. 10.1111/febs. 14099

Ward JJ, Sodhi JS, McGuffin LJ, Buxton BF, and Jones DT. 2004. Prediction and functional analysis of native disorder in proteins from the three kingdoms of life. J Mol Biol 337:635-645. 10.1016/j.jmb.2004.02.002

Zambrano R, Conchillo-Sole O, Iglesias V, Illa R, Rousseau F, Schymkowitz J, Sabate R, Daura X, and Ventura S. 2015. PrionW: a server to identify proteins containing glutamine/asparagine rich prion-like domains and their amyloid cores. Nucleic Acids Res 43:W331-337. 10.1093/nar/gkv490

Zhang K, Liu Q, Shen D, Tai H, Fu H, Liu S, Wang Z, Shi J, Ding Q, Li X, Liu M, Cui L, and Zhang X. 2018. Genetic analysis of TIA1 gene in Chinese patients with amyotrophic lateral sclerosis. Neurobiol Aging 67:201 e209-201 e210. 10.1016/j.neurobiolaging.2018.03.020 


\section{Supplementary Materials}

467 Data S1 and Data S2: FASTA-format files of the protein sequences for the Pub1/Tia1 and 468 Nam8/Ngr1 families used for phylogenetic analysis.

Table S1: BLASTP output showing the most similar proteins to Pub1, Ngr1 and Nam8 in the yeast proteome.

472

Figure S1: Phylogenetic tree with additional paralogs. This is made using RaxML (Stamatakis 2015), as described in Methods, and drawn using iTOL (Letunic \& Bork 2019). The bootstrap values are colour-coded with gradient colouring for 0.5 to 1.0 , ranging from red for 0.5 to green for 1.0, with $<0.5$ black. The UniProt (UniProt 2019) accession numbers for proteins are used if

477 available, but otherwise the protein identifier from the OrthoDB database is used (Kriventseva et 478 al. 2019). Pub1/Tia1/Tiar orthologs are coloured purple, Nam8 orthologs blue and Ngr1 green. The identifiers of additional paralogs are coloured yellow. The circles of annotations are as follows in order from inside to outside:-

(i) PLAAC (Lancaster et al. 2014) LLR prion-like composition score (red);

482

(ii) percentage of residues annotated as disordered by IUPred2a (Dosztanyi 2018) (blue).

485 family used to annotate the phylogenetic trees. 
488 Table S3: Bioinformatic data for Nam8 family (Excel file). Bioinformatics data for the Nam8

489 family used to annotate the phylogenetic trees.

490

491 Figure Legends

492 Figure 1: Phylogenetic tree of Pub1 and Nam8 in Saccharomycetes. This is made using PhyML

493 (Guindon et al. 2005), as described in Methods, and drawn using Evolview (Subramanian et al.

494 2019). The UniProt (UniProt 2019) accession numbers for proteins are used. The aBayes support

495 values are colour-coded black for $<0.5$, green for $0.5-0.65$, magenta for $0.65-0.8$ and cyan for 0.8 -

496 1.0. The PLAAC (Lancaster et al. 2014) log likelihood ratio (LLR) prion-like composition scores

497 are depicted as a histogram around the circumference of the tree. There is an axis of for the LLR

498 values at the start of the circle. The UniProt accession number for Pub1 is P32588, for Nam8 it is

499 Q00539 and for Ngr1 it is P32831. These accession numbers are highlighted in gold.

500

501 Figure 2: Phylogenetic tree of Pub1 / Tia1 and Nam8 / Ngr1 for the eukaryotic domain. This

502 is made using PhyML (Guindon et al. 2005), as described in Methods, and drawn using Evolview

503 (Subramanian et al. 2019). The aBayes support values are colour-coded black for $<0.5$, green for

504 0.5-0.65, magenta for 0.65-0.8 and cyan for 0.8-1.0. The UniProt (UniProt 2019) accession

505 numbers for proteins are used if available, but otherwise the protein identifier from the OrthoDB

506 database is used (Kriventseva et al. 2019). Pub1/Tia1/Tiar orthologs are coloured purple, Nam8

507 orthologs blue and Ngr1 green. The circles of annotations are as follows in order from inside to 508 outside:-

509 (i) labelling of Mammalian and Saccharomycetes proteins (green and brown respectively); 
510 (ii) colour-coded bars for the Pub1 Saccharomycetes clade (magenta), mammalian Tia1

511 (bright green), mammalian Tiar (gold), Saccharomycetes Nam8/Ngr1 (dark purple),

512 mammalian Nam8 (dark blue);

513 (iii) PLAAC (Lancaster et al. 2014) LLR prion-like composition score (red);

514 (iv) percentage of residues annotated as disordered by IUPred2a (Dosztanyi 2018) (blue);

515 (v) percentage of residues annotated as disordered by DISOPRED3 (Ward et al. 2004) (teal);

516 (vi) number of RRM domains in each protein (dark green);

517 (vii) number of prion-like domains in each protein, using the threshold of $>0.0$ (pink);

518 (viii) number of prion-like domains in each protein, using the threshold of $\geq 15.0$ (gold);

519 (ix) $\log 10$ of the fLPS binomial P-value for compositional bias for $\mathrm{Q}$ and $\mathrm{N}$ residues (Harrison 520 2017) (dark grey).

521 The data for this figure are in Tables S2 and S3.

523 Figure 3: Scores for prion-like composition. (a) Distribution of prion-like PLAAC log-

524 likelihood ratio scores (Lancaster et al. 2014) corresponding to the data in Figure 2, but with purple 525 colour for Pub1/Tia1/Tiar, and Nam8 and Ngr1 are merged into one set labelled blue. The intervals 526 of prion-like scores are labelled with the value of their midpoints, i.e., 5 for $0-10$, etc.

527 (b) Scatter plot of intrinsic disorder versus prion-like scores with the same sequence colour-coding, 528 with linear regression lines fitted. The P-values are two-tailed.

530 Figure 4: Numbers of RNA-binding and prion-like domains. (a) The number of RNA-binding

531 domains for the proteins in Figure 2 (sequence colour-coding same as Figure 3). 
532 (b) and (c) The number of prion-like domains for the proteins in Figure 2 (sequence colour533 coding same as Figure 3), for a PLAAC LLR score of $>0.0$ and $\geq 15$. 


\section{Figure 1}

Figure 1: Phylogenetic tree of Pub1 and Nam8 in Saccharomycetes.

This is made using PhyML (Guindon et al. 2005) , as described in Methods, and drawn using Evolview (Subramanian et al. 2019) . The UniProt (UniProt 2019) names for proteins are used. The support values are colour-coded green for $0.5-0.65$, magenta for $0.65-0.8$ and cyan for 0.8-1.0. The PLAAC (Lancaster et al. 2014) log likelihood ratio (LLR) prion-like composition scores are depicted as a histogram around the circumference of the tree. The UniProt identifier for Pub1 is P32588, for Nam8 it is Q00539 and for Ngr1 it is P32831. 


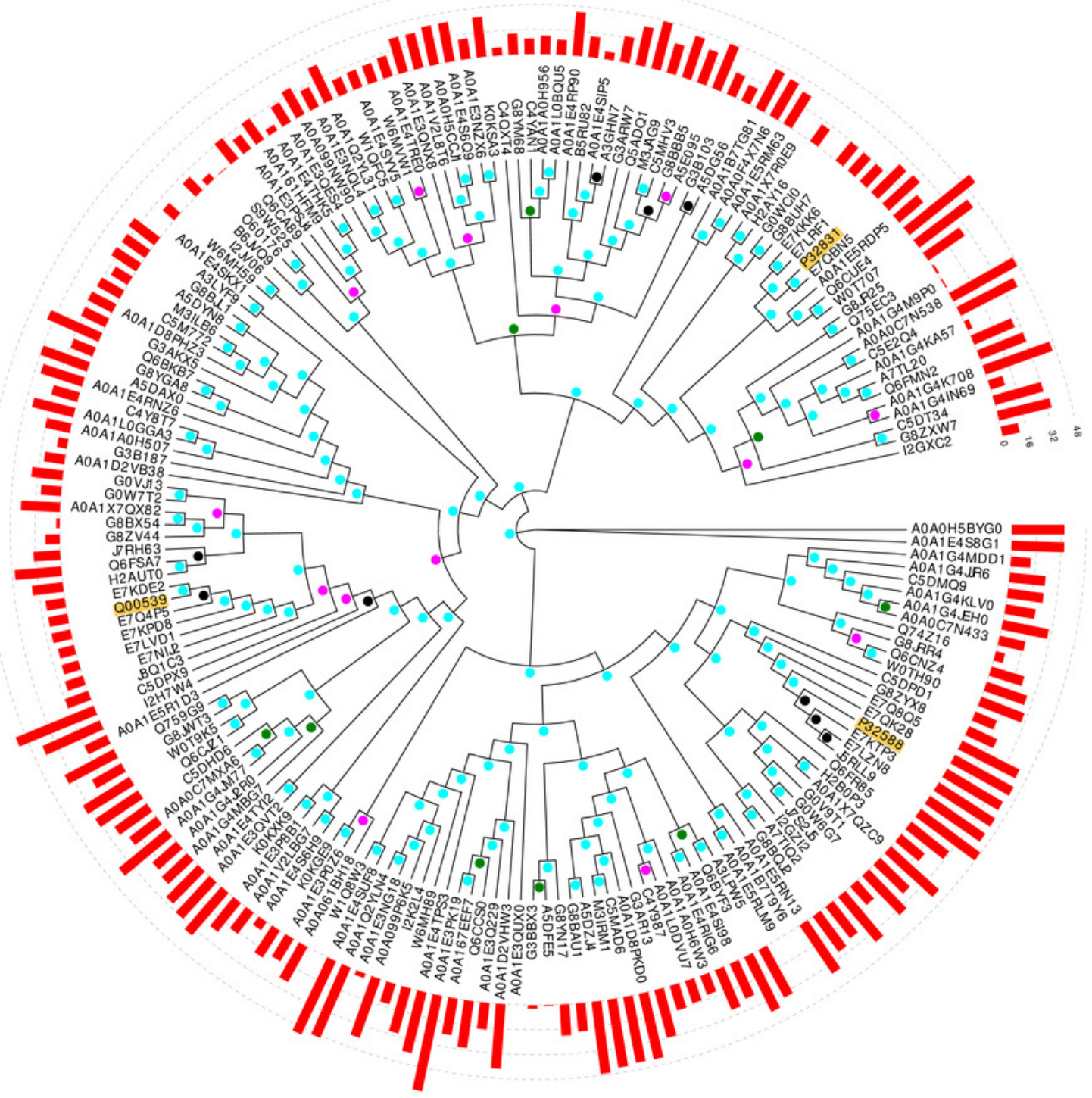




\section{Figure 2}

Figure 2: Phylogenetic tree of Pub1 / Tia1 and Nam8 / Ngr1 for the eukaryotic domain.

This is made using PhyML (Guindon et al. 2005), as described in Methods, and drawn using Evolview (Subramanian et al. 2019) . The support values are colour-coded green for 0.5-0.65, magenta for 0.65-0.8 and cyan for 0.8-1.0. The UniProt (UniProt 2019) names for proteins are used if available, but otherwise the protein identifier from the OrthoDB database is used (Kriventseva et al. 2019) . Pub1/Tia1/Tiar orthologs are coloured purple, Nam8 orthologs blue and Ngr1 green. The circles of annotations are as follows in order from inside to outside:- <!-[if !supportLists]-->(i) <!--[endif]-->labelling of Mammalian and Saccharomycetes proteins (green and brown respectively); <!--[if !supportLists]-->(ii) <!--[endif]-->colour-coded bars for the Pub1 Saccharomycetes clade (magenta), mammalian Tia1 (bright green), mammalian Tiar (gold), Saccharomycetes Nam8/Ngr1 (dark purple), mammalian Nam8 (dark blue); <!--[if !supportLists]-->(iii) <!--[endif]-->PLAAC (Lancaster et al. 2014) LLR prion-like composition score (red); <!--[if !supportLists]-->(iv) <!--[endif]-->percentage of residues annotated as disordered by IUPred2a (Dosztanyi 2018) (blue); <!--[if !supportLists]-->(v) <!--[endif]->number of RRM domains in each protein (dark green); <!--[if !supportLists]-->(vi) <!-[endif]--> \& (vii) number of prion-like domains in each protein, using the threshold of $\geq 0.0$ (pink), and $\geq 15.0$ (gold). 


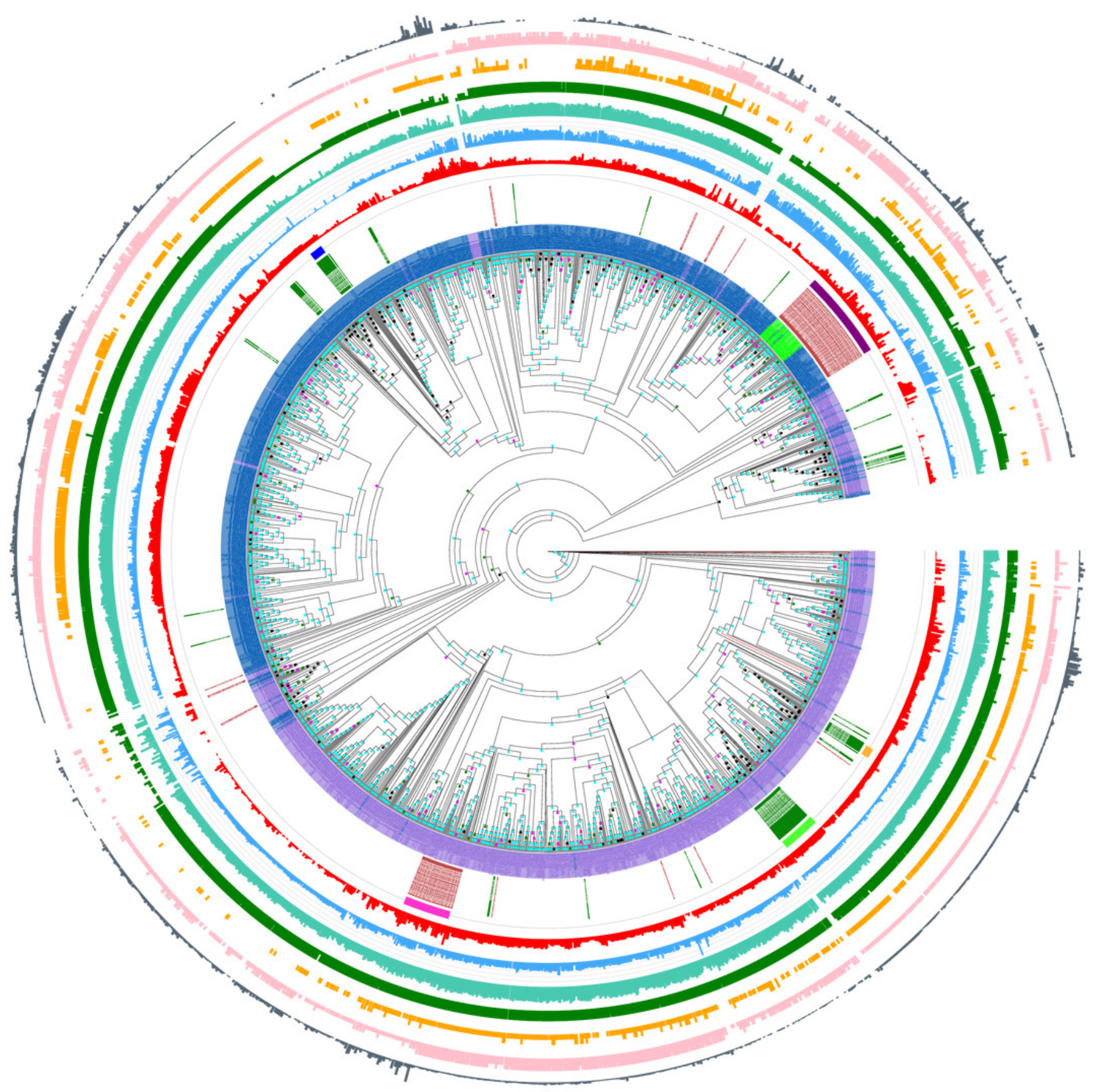




\section{Figure 3}

Figure 3: Scores for prion-like composition.

(a) Distribution of prion-like PLAAC log-likelihood ratio scores (Lancaster et al. 2014) corresponding to the data in Figure 2, with the same sequence colour-coding except Nam8 and Ngr1 are merged into one set labelled blue (Pub1/Tia1/Tiar and Nam8+Ngr1). The intervals of prion-like scores are labelled with the value of their midpoints, i.e., 5 for 0-10, etc. (b) Scatter plot of intrinsic disorder versus prion-like scores with the same sequence colour-coding, with linear regression lines fitted.

(A)

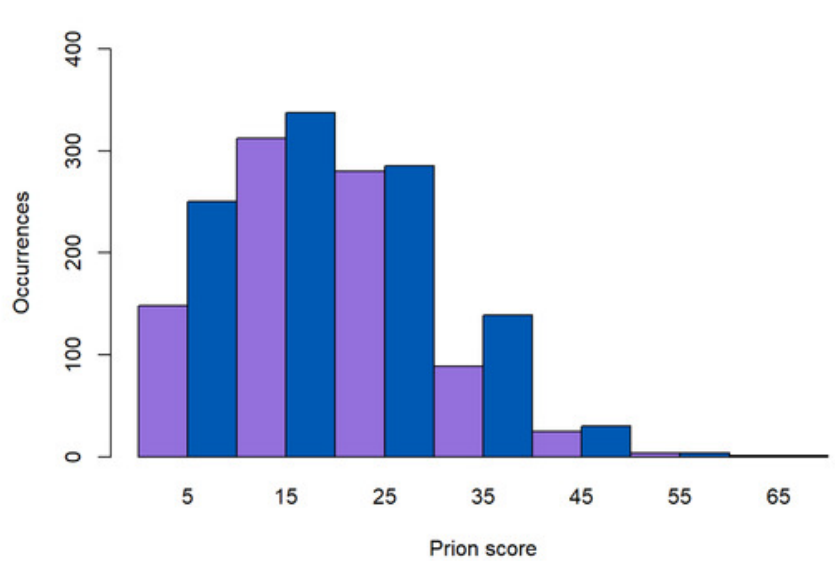

(B)

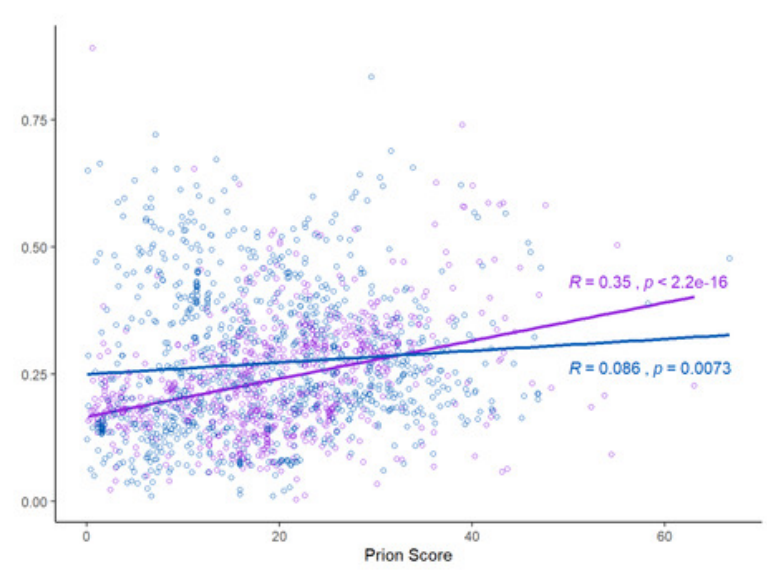




\section{Figure 4}

Figure 4: Numbers of RNA-binding and prion-like domains.

(a) The number of RNA-binding domains for the proteins in Figure 2 (sequence colour-coding same as Figure 2). The intervals of prion-like scores are labelled with the value of their midpoints, i.e., 5 for 0-10, etc. (b) and (c) The number of prion-like domains for the proteins in Figure 2 (sequence colour-coding same as Figure 2), for a PLAAC LLR score of $\geq 0.0$ and 215.0. The intervals of prion-like scores are labelled with the value of their midpoints, i.e., 5 for $0-10$, etc.

(A)

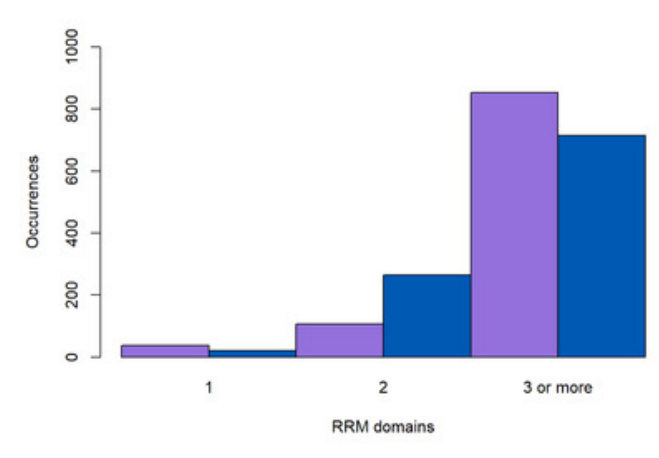

(B)

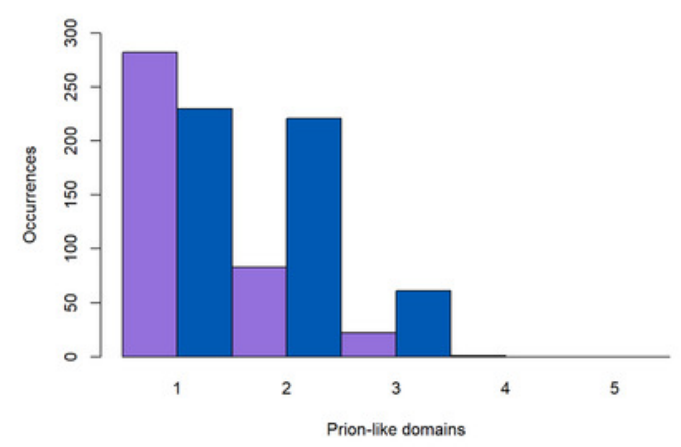

(C)

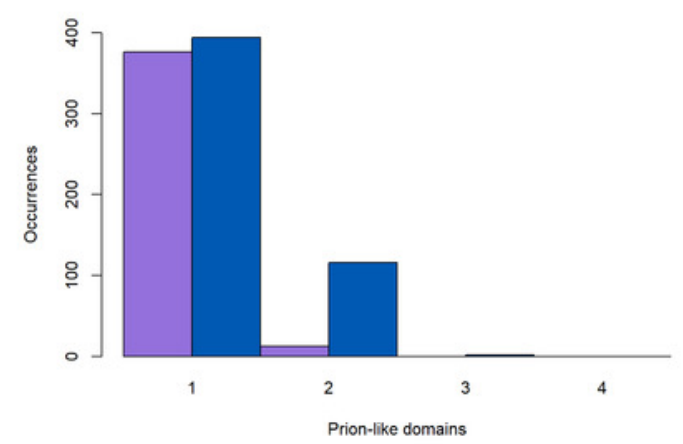

